\title{
IN BED WITH MADONNA. GEDANKEN ZUR ANALYSE VON VIDEOCLIPS AUS MEDIENTHEORETISCHER SICHT ${ }^{1}$
}

\author{
Dietrich Helms
}

Der Versuch, dem Phänomen Videoclip in der wissenschaftlichen Beschreibung möglichst gerecht zu werden, hat in der musikwissenschaftlichen Literatur zur Konstruktion immer komplexerer Kategorien- bzw. Variablensysteme geführt (vgl. z.B. Pape/Thomsen 1997, Bullerjahn 2001, Altrogge 2000). Einige vorgeschlagene Systeme sind inzwischen so komplex, dass sie, wenn sie tatsächlich auf ein konkretes Objekt angewandt werden sollen, durch ihre Komplexität und die Unvereinbarkeit ihrer Kategorien unweigerlich wieder das erzeugen, was sie eigentlich einschränken sollten: Kontingenz (vgl. auch den Beitrag v. Helmut Rösing in diesem Band). Die musikwissenschaftliche Videoclipanalyse ist an einem Punkt angelangt, an dem sich die Physik zu Beginn des vergangenen Jahrhunderts befand: Die Atomisierung des Gegenstands in immer kleinere Teile muss mit jedem weiteren Schritt der Differenzierung zu der Erkenntnis führen, dass das, was beobachtet wird, ein Produkt der Beobachtung ist. Die Gestalttheorie, noch vierzig Jahre älter als die Heisenbergsche Unschärferelation (1927), hat uns gelehrt, dass ein Gegenstand nicht zureichend durch die Beschreibung seiner Teile definiert wird. Christian von Ehrenfels' Theorie der Übersummativität (vgl. Ehrenfels 1967: 13ff.) besagt, dass ein Gegenstand immer mehr ist als die Summe seiner Teile - und es ist gerade dieses Mehr, welches den Gegenstand ausmacht. So demonstriert eine quasi atomisierende Analyse eines Clips zwar eindrucksvoll die Möglichkeiten der Technik, sie kommt in ihrem maximalem Aufwand und dem Bemühen, den Überblick zu behalten, dem Ganzen jedoch nicht näher - auch in dieser Erfahrung ist die Physik der Musikwissenschaft einen Quantensprung voraus.

1 In Bed with Madonna ist der Titel eines Konzertfilms mit und über Madonna (USA 1991, Regie: Alek Keshishian), kein Videoclip. Der Leser möge diese Ungenauigkeit zugunsten der Anschaulichkeit des Titels verzeihen. 
Hermeneutik geht von der Annahme aus, dass es einen Gegenstand gibt, ein unveränderliches Objekt, das etwas bedeutet, sei es an sich durch seine objektive Qualität, oder, spätestens seit Hans-Georg Gadamer (1986), auch durch die Bemühungen des Beobachters, sich auf der Grundlage des eigenen, subjektiven Wissens dem Objekt anzunähern. Die Unmöglichkeit der wahren Erkenntnis des Objekts, die Gadamers Hermeneutik, aber auch z.B. die Rezeptionsästhetik der so genannten Konstanzer Schule (vgl. z.B. Jauß 1991) feststellten, führt in letzter Konsequenz dazu, dass das Objekt, das erkannt werden soll, aus dem Blickfeld gerät. Es initiiert Sinn, der doch nicht sein eigener sein kann, da dieser ja nicht zu erkennen ist. Es sendet Zeichen, ohne per se aus Zeichen zu bestehen. Gebraucht wird die Entität Text bzw. Werk - denkt man den Ansatz der Rezeptionsästhetik konsequent zu Ende - nur noch, um Aussagen über Wirkungen oder Rezeptionen in Beziehung setzen zu können, die, da sie das Werk ohnehin nicht beschreiben können, sonst in völliger »subjektiver « Beliebigkeit auseinander streben würden (vgl. die Kritik von Dahlhaus 1977: 240f.). Das Denken in SubjektObjekt-Differenzen in den Kunstwissenschaften ist mit Hermeneutik und Rezeptionsästhetik der 1960er und 1970er Jahre an einer Grenze angekommen, die, obwohl sie so lange bekannt ist, von der Musikwissenschaft im Gegensatz z.B. zu den Medienwissenschaften bisher selten überschritten wurde.

Gadamer und die Konstanzer Schule geben die Richtung des Weges jenseits der Grenze vor: Bedeutung ist abhängig vom Rezipienten. Diesem die volle Verantwortung zuzuschreiben, nicht nur für die Bedeutung, sondern auch für den Gegenstand selbst (und damit auch für die Wirkung im Sinne der Rezeptionsästhetik), wagt den Schritt über die Demarkationslinie, weg vom Denken in Relationen von Subjekten und Objekten. Nicht der Gegenstand macht dort den Sinn in seiner Konfrontation mit dem Wahrnehmenden. Was zuvor als Objekt aufgefasst wurde, ist jetzt ein Produkt des Prozesses der Erzeugung von Sinn durch einen Beobachter, dessen grundlegende Operation die Unterscheidung von System und Umwelt ist (vgl. zur Beschreibung dieses Begriffs von Sinn Luhmann 1984: 92ff.). ${ }^{2}$ Sinn entsteht, indem ein psychisches oder ein soziales System einer aktuellen Information

2 Diese Grundlage der Theorie sozialer Systeme lässt sich auch biologisch begründen. Die aktuelle Neurobiologie sieht nicht mehr Erkenntnis als Gipfelpunkt des konvertierenden Teils der neuronalen Reizverarbeitung, sondern Verhalten (Roth 1997: 151). Es kommt nicht darauf an, dass das Lebewesen seine Umwelt erkennt, sondern dass es auf eine Unterscheidung in seiner Umwelt mit geeignetem Anschlussverhalten reagiert. Dazu muss es der Aktualität der Information Potenzialität, d.h. Möglichkeiten des Verhaltens, zuordnen können, also in Sinnsystemen operieren. 
Anschlussmöglichkeiten zuweist, d.h. aus der Unterscheidung von Aktualität und Potentialität (Luhmann 1999: 174). Dinge, Zeit und Kommunikation entstehen erst in diesem Prozess der Differenzierung von Aktuellem und Möglichen und sind Dimensionen des Sinns: die Unterscheidung von dem Einen und dem Anderem, dem Vorher und dem Nachher, der Ego- und der Alterperspektive. Das Sinnsystem, das mit dem Begriff »Videoclip« symbolisch generalisiert wird, ist in dieses Koordinatensystem einzuordnen.

Wir meinen, einen Videoclip als Ding beschreiben zu können, weil wir erwarten, dass er auch in Zukunft das Eine und nicht das Andere sein wird. Der Beobachter geht davon aus, dass sich die sachliche Dimension des Sinnsystems »Videoclip « in der Zukunft nicht verändern wird. Wenn wir jedoch von Wirkung und Bedeutung als immanenten Eigenschaften des Videoclips sprechen, beziehen wir uns auf eine Sinndimension, die ein Ding per se nicht haben kann: ein Adressat Ego sieht in dem Video die Handlungen eines Alters und versteht diese Handlungen unter Umständen als Mitteilung, d.h. als den Versuch sein Verhalten zu orientieren (womit der Text eine Bedeutung für Ego und eine Wirkung für einen Beobachter Egos bekommt). Diese soziale Dimension des Sinns läuft der Vorstellung des Dingseins des Clips entgegen, denn die Differenz von Ego und Alter, aus der soziale Systeme entstehen, kann niemals durch die Dimension Zeit hindurch gleich bleiben. Sie ist gerade gekennzeichnet durch Veränderung. Wenn auch die Handlungen des Mitteilenden Alter auf Magnetband gebannt und wiederholbar werden: Ego verändert sich und damit auch die Art, wie er das beobachtete Verhalten Alters als Handlungen oder Kommunikation auffasst.

Diese Gegenläufigkeit der sachlichen und der sozialen Dimension von Sinn in Bezug auf die Dimension Zeit ist kennzeichnend für Verbreitungsmedien. Will man ein Verbreitungsmedium wie z.B. einen Videoclip mit Hilfe eines Dingschemas beschreiben, entstehen aus dieser Differenz epistemologische Probleme. Mit dem Begriff des Werks hat die Hermeneutik versucht, die Spannung im Paradigma der Kunstmusik zwischen unveränderlichem Ding und sich ständig wandelnder sozialer Kopplung zu überbrücken. Das wahrnehmbare Dingsein des musikalischen Werks, das Notenblatt oder auch die klingende Musik, wird nur als Reflex des Werkes aufgefasst. Es ist Ausdruck seines veränderlichen Seins. Als das eigentliche Ding wird das unveränderliche Sein des Musikstücks angesehen, die platonische Idee, die im Bereich der Metaphysik oder auch in der individuellen Psychologie des Komponisten an einem bestimmten Punkt seiner Biographie verortet wird. Damit wird nicht nur die sachliche, sondern auch die soziale Dimension als einzig wahre Botschaft des Komponisten zeitlich fixiert, die fixierte Form aber gleichzeitig der endgültigen Fassbarkeit entzogen. D.h. eine Hermeneutik, die nach 
Erkenntnis der Idee bzw. des Werkes strebt, muss die Erkennbarkeit des Werkes negieren, um den Dingcharakter zu erhalten, dessen Erkenntnis angestrebt wird (vgl. zur Idee als Dingschema Luhmann 1984: 98f.).

Moderne Verbreitungsmedien wie der Videoclip oder auch schon die 100 Jahre ältere Schallaufzeichnung eignen sich allerdings kaum für eine werkimmanente, hermeneutische Betrachtungsweise, da 1. das »Material« des Videoclips, an dem sein Dingsein bestimmt werden könnte, nicht festzulegen ist und 2. der Clip als Medium kein Verhalten provoziert, dass wir als Interpretation im Sinne des Systems der Kunstmusik beschreiben würden. Ich habe diese Gedanken bereits ausführlich am Beispiel des Phänomens »Sound« und des Mediums der Schallaufzeichnung erläutert (Helms 2003) und möchte daher an dieser Stelle nur kurz darauf eingehen. Die Vorstellung, dass Musik etwas anderes sein könnte als Handlung und Kommunikation, ist eng mit der Erfindung der Notenschrift und vor allem dem ersten Medium massenhafter Verbreitung von Musik, dem Notendruck, verknüpft. Noten sind Beschreibungen von Handlungen eines Musikers. Durch sie wird ein sozial- und zeitbedingtes Sinnsystem durch ein zeitloses, sachliches Sinnsystem ersetzt, ein vermeintliches Ding, das den Musiker zum Komponisten, zum Schöpfer macht. Dieses Ding kann durch Handlungen von Musikern, aber auch von Musikwissenschaftlern beschrieben werden und damit wiederum in ein sozial- und zeitbedingtes Sinnsystem (die Handlung des Musizierens) bzw. in ein weiteres dingliches Verbreitungsmedium (die schriftsprachliche Interpretation) umgesetzt werden. Eine Handlung als Beschreibung des Gegenstands »Noten« wird im gängigen Paradigma nicht als übereinstimmend mit der Handlung des Komponisten angesehen. Diese bleibt unbeobachtbar hinter den Noten verborgen und ermöglicht damit ihre Verdinglichung zum Werk bzw. der Idee. Die Unmöglichkeit der Beobachtung der ursprünglichen Handlung durch das Verbreitungsmedium Notation hält die Dynamik der ständigen Interpretation in Gang, die so lange zu weiteren Interpretationen führen muss, wie Musiker und Musikwissenschaftler ihr

Anschlussverhalten an das Medium Notation als Anschluss an ein Werk verstehen: »Der Glaube an den Gehalt der Werke, der zu entschlüsseln wäre, folgt [...] allein der imperativ wirkenden Mediengrammatik, die durch Gestaltungsoptionen Glaubensbekenntnisse verfügt« (Schläbitz 1997: 24).

Ganz anders verhält es sich dagegen mit den neueren Verbreitungsmedien der Musik. Schallaufzeichnung und Videoclip zeichnen sich durch die Vorstellung des Beobachters aus, dass sie Handlungen »wiedergeben«. Das Video gibt vor - augenfälliger als die reine Schallaufzeichnung -, MusikMachen beobachtbar zu machen. Dagegen entzieht sich dem Beobachter das 
Ding, das Material der »Aufzeichnung « (vgl. Großmann 1997: 69f.). Videoband, DVD, Schallplatte oder CD sind nur von Maschinen zu beschreiben und zu lesen. Sinn macht eine CD oder DVD für den Beobachter der »äußeren« Form nur als Entität; ihre Teile, die vom Laser eingebrannten Vertiefungen in der Trägerschicht, enthalten keine Informationen, die für psychische Systeme zugänglich wären, es sei denn, eine Maschine übernähme eine weitere Stufe der Beschreibung. Noch deutlicher fällt diese Entdinglichung im Fall von Rundfunk und Fernsehübertragungen aus: Die Materialität der CD bzw. des Videobands existiert hier nur noch in der Vorstellung des Beobachters, ist für ihn jedoch nicht mehr als Konkretum zu erfahren. Er schließt sie aus der Möglichkeit der Wiederholbarkeit des Songs oder des Videos. Ähnliches gilt für die Speicherung von Bildern und Musik auf den Festplatten von Computern.

Die Unzugänglichkeit der dinglichen Gestalt des Mediums lässt den Eindruck entstehen, die am Bildschirm oder Lautsprecher beobachtete Handlung sei identisch mit der Handlung des Musikers, die das Medium erzeugte. Es entsteht die Vorstellung von einer »Aufzeichnung «. Eine als Interpretation zu bezeichnende Anschlusshandlung des Beobachters erübrigt sich hierdurch. Die im System des Mediums Noten Interpretation genannte Handlung schrumpft zusammen auf das Drücken der »play«-Taste des Videorecorders oder der Programmwahltaste des Fernsehers. Wenn Madonna »I'm A Material Girl« singt und wir sie im Videoclip auch optisch beim Singen beobachten können, gibt es keinen Grund anzunehmen, dass das Stück eigentlich anders klingen, der Vorgang des Musizierens anders dargestellt werden sollte oder könnte. Mit der musikalischen Interpretation erübrigt sich auch die sprachliche Interpretation durch die Musikwissenschaft, das Bemühen um die Erkenntnis der wahren Gestalt oder der wahren Botschaft des verborgenen Werks. Angesichts eines Mediums, das keine Interpretation benötigt, um verstanden zu werden, bleibt dem Wissenschaftler kaum mehr als die nacherzählende Beschreibung der eigenen Sicht auf die offensichtlichen Handlungen dieser künstlichen Wesen namens Aphex Twin oder Madonna.

Mit der dinglichen Gestalt des Videoclips verschwinden auch die Handlungen, die zur Produktion der Form des Mediums führen, aus der Aufmerksamkeit des Beobachters. Noten lenken die Aufmerksamkeit auf das Komponieren. Dagegen treten beim Videoclip die Handlungen von Komponisten, Textdichtern, Choreographen, Toningenieuren, A\&R-Managern, Drehbuchund Sciptschreibern, Regisseuren, Cuttern, Kameramännern, Beleuchtern, Dekorateuren, Computeranimateuren, Kostümbildnern, Maskenbildnern, Imageberatern, Kulissenbauern, Programmplanern, Marketingmanagern, Trendscouts und vielen mehr, ja selbst die tatsächlichen Handlungen der 
Musiker selbst völlig in den Hintergrund. Sie verschwinden in ihrer Einmaligkeit hinter den im Clip beobachtbaren, wiederholbaren Handlungen einer virtuellen Entität, die den Namen der Band oder des Interpreten trägt. Die Wiederholbarkeit der sachlichen Dimension des Mediums lässt den Eindruck eines einheitlichen Willens hinter den beobachteten Handlungen entstehen - so fragen Journalisten die Mitglieder von Bands in Interviews: »Was wollt ihr mit dem neuen Video aussagen?« und auch die Musikwissenschaft sucht noch oft nach einer, nach der Bedeutung. Der Gedanke, dass jeder Beteiligte eine andere Vorstellung von seinem Tun hatte und selbst der eigenwilligste, durchsetzungsfähigste Popstar nur Teil des Systems, nicht jedoch das System selbst ist, wird für den Beobachter durch das Medium verhüllt. Massenmedien können keinen allmächtigen Schöpfer, keinen einheitlichen Schöpferwillen haben. Der Name einer Band oder einer Sängerin ist nicht mehr als eine symbolische Generalisierung für ein hochkomplexes soziales System. Die Suche nach einer »Idee hinter« dem Beobachteten kann, angesichts der großen Zahl der an der Entstehung des Clips Beteiligten, nur zur Suche nach dem kleinsten gemeinsamen Nenner und damit zur Feststellung der Banalität oder von Banalitäten führen.

Im Gegensatz zur Musikkritik, die im System der Kunstmusik die Kommunikation zwischen Musiker und Zuhörer beschreibt, fehlt dem Beobachter von ausschließlich durch Schallaufzeichnung und Videoclip verbreiteter Musik die Möglichkeit einer zweiten Interpretation (die auch die eigene sein kann) als tertium comparationis. Aussagen über beobachtete Handlungen von Musikern in Medien der Schall- und Bildaufzeichnung haben immer den Anschein von Subjektivität, da zur Objektivität das Werk als fixes, wenn auch paradoxerweise nicht erkennbares Objekt fehlt, und den Anschein von Irrelevanz, da immanente, sprachliche Interpretation in Abwesenheit des Werkes und der Offensichtlichkeit der Handlungen nicht notwendig, musikalische Interpretation nicht möglich ist. Dies ist vermutlich ein Grund, warum sich Musikwissenschaft, wenn sie sich mit Schallaufzeichnungen und Videoclips beschäftigt, bevorzugt deren Wirkung und den daraus resultierenden Funktionen zuwendet. Als Vergleichsobjekte dienen dem Beobachter hier mehrere »Individuen« oder mehrere »soziale Gruppen«, deren Handlungen in Beziehung zu einer Schall- oder Bildaufzeichnung in Differenz zueinander gesetzt werden. Für die Untersuchung von Wirkungen ist das Dingschema ebenso konsequent vorauszusetzen wie für die Interpretation von Bedeutungen. Der wissenschaftliche Beobachter bestimmt die Wirkung eines Clips durch Beobachtung von Verhaltensänderungen ${ }^{3}$ der Probanden bei der

3 Als Verhaltensänderungen verstehe ich hier auch z.B. Änderungen von Verhalten der Anzeigen medizinischer Diagnosegeräte (z.B. Blutdruckmessgeräte, 
Wahrnehmung des Clips. Die Begründung der Verhaltensänderung durch den Bezug auf den Clip ist nur möglich, wenn man von seiner grundsätzlichen Unveränderbarkeit ausgeht. Fasst man Wahrnehmung jedoch nicht als passive Rezeption, sondern als Operieren in Sinnsystemen auf, verliert die Wirkungsforschung unweigerlich ihren Archimedischen Punkt. ${ }^{4}$ Der Videoclip ist nicht nur eine Zeit unabhängige Differenz zwischen dem Einen und dem Anderen, sondern enthält eben auch die Zeit abhängige, veränderliche Differenz zwischen Ego und Alter. Es klingt banal: Der Beobachter erster Ordnung, dessen Verhalten der wissenschaftliche Beobachter (zweiter Ordnung) beschreibt, orientiert sein Verhalten nicht an der Magnetisierung des Videobandes - diese mag gleich bleiben. Er ändert sein Verhalten auch nicht aufgrund der Gegenstände, die er auf dem Bildschirm sehen kann, während der Clip läuft - die sachliche Dimension ist lediglich wichtig für das Erkennen von gleich oder verschieden. Er ändert sein Verhalten, weil er Handlungen, die er an seinem Gegenüber beobachtet, und Gegenstände, die er als Ergebnis von dessen Handlungen bzw. als potentielle Objekte der Interaktion erkennt, zu sich selbst in Beziehung setzt und sie damit in Kommunikation verwandelt. Das gilt auch für Clips ohne menschliche Darsteller und abstrakte Filme, denn wie bei der Schallaufzeichnung impliziert das Beobachtete die Handlung eines Alters. Man hört oder sieht nicht ein Ding, sondern man hört oder sieht einem Gegenüber beim Musik- und Filmemachen zu und vermutet in dessen Handeln einen Mitteilungswillen. Kommunikation entsteht, wenn der Wahrnehmende, der Zuschauer des Videoclips, die Möglichkeit erkennt, dass er auf das beobachtete Mitteilungsverhalten mit einem bestimmten Anschlussverhalten reagieren könnte (Luhmann 1996: 14). Eine Mitteilung Alters existiert nur durch das Verstehen Egos. Das Video wäre kein Medium und hätte weder »Bedeutung« noch »Wirkung« ohne diese strukturelle Koppelung. Es ist daher auch nicht zu beschreiben ohne Be-

Geräte zur Messung des Hautwiderstands, EEG und EKG) und sprachliche Beschreibungen psychischer Zustände.

4 Die exakte Wiederholung des Versuchsaufbaus mit unterschiedlichen Probanden gilt nur für die Perspektive des wissenschaftlichen Beobachters, d.h. des Beobachters zweiter Ordnung. Der Wissenschaftler beobachtet ja die Interaktionen von Clip und Proband auf der Grundlage der Handlungen des Probanden. Die Interaktion zwischen den auf dem Clip zu beobachtenden Handlungen und dem Wissenschaftler werden vernachlässigt. Das gilt jedoch nicht für die Probanden, von denen jeder zu jedem unterschiedlichen Zeitpunkt, in jedem unterschiedlichen sozialen und lokalen Kontext andere Mitteilungen beobachtet, die ein je anders motiviertes Anschlussverhalten auslösen. Der Wissenschaftler kann demnach nicht gleiche Wirkungen beobachten, sondern nur gleiches Verhalten. Er hat keinen Zugriff auf die Kausalität des beobachteten Verhaltens, wie sie sich für das beobachtete psychische System ergibt. 
schreibung der vom zeitlichen, räumlichen und sozialen Kontext Egos abhängigen Ego-Alter-Perspektive bzw. ohne dass diese in die Beschreibung eingeschrieben wäre.

Bereits mit der Entwicklung des ersten Verbreitungsmediums der Musik, der Notation und vor allem mit ihrer Weiterentwicklung zum Massenmedium Notendruck, entstanden Verständnisprobleme. ${ }^{5}$ Die Abwesenheit des Mitteilenden Alter, der jetzt Komponist genannt wurde, lässt den Adressaten Ego, den Musiker, im Ungewissen, ob sein Anschlussverhalten »richtig « ist. Es fehlt ihm zur Gewissheit die Rückkoppelung mit der Reaktion Alters. Diesem Verstehensproblem wurde begegnet durch immer stärkere Ausdifferenzierung des Mediums, aber auch durch die Entwicklung weiterer Verstehensmedien, d.h. durch Vereinheitlichung und Verfeinerung der Notenschrift, Einführung des Mediums Sprache in Vortragsbezeichnungen, Titeln, Programmen sowie des Mediums Bild in Illustrationen bzw. Titelkupfern. Zudem entwickelten sich sekundäre Verstehensmedien ${ }^{6}$ : An die Stelle des abwesenden Komponisten trat der Zuhörer, an dessen Verhalten sich der Musiker orientieren konnte. In dem Maße, wie sich Akademien und Salons in Konzerte verwandelten und die direkte Koppelung zwischen Musiker und Zuhörer erschwerten, waren weitere sekundäre Verstehensmedien notwendig, die den abnehmenden Kontakt des Hörers mit dem Musiker durch Koppelung der Hörer untereinander ersetzte: das Gespräch im Foyer, die Musikkritik. Der Notendruck führte zur endgültigen Spaltung des Systems des Musikmachens in die geschlossenen Systeme von Komponieren und Musizieren. Und mit der Erfindung der Schallaufzeichnung schließlich zerfiel endgültig die Einheit von Musizieren und Hören in zwei geschlossene Systeme. Der Musiker des neuen Massenmediums hat seitdem keine Möglichkeit mehr, die Auswirkungen von Änderungen seines Verhaltens auf das Verhalten seiner Adressaten zu beobachten. Er dreht sich bei der Aufnahme im Studio gleichsam um sich selbst - zumal die Mehrspurtechnik auch die direkte Kommunikation der Musiker untereinander im Medium Musik unterbindet und auf das Medium

5 Videoclips als Medien sind keine Speicher, die eine Information über zeitliche und räumliche Distanz unverändert transportieren - die Metapher führt in die Irre -, sie sind vielmehr in der Definition Luhmanns »diejenigen evolutionären Errungenschaften, die an jenen Bruchstellen der Kommunikation ansetzen und funktionsgenau dazu dienen, Unwahrscheinliches in Wahrscheinliches zu transformieren « (Luhmann 1984: 220), d.h. die die Probleme des Zustandekommens von Kommunikation, nämlich Verstehen, Erreichbarkeit und Erfolg, begrenzen. Kommunikation kommt zustande mit der Zustandsänderung des Adressaten Ego durch die Handlung Alters. Verstehen kommt zustande, wenn der Mitteilende das Verhalten des Adressaten als Anschlusshandlung an seine Mitteilung erkennt.

6 Ich verwende den Begriff sekundäre Verstehensmedien für solche Medien, die Kommunikation über Kommunikation zum Gegenstand haben. 
Sprache zurückgegriffen werden muss, um Verhalten wechselseitig zu orientieren.

Dass seine Mitteilung vom Hörer verstanden wurde, kann der Musiker außerhalb des Konzerts nur durch Koppelung mit anderen sozialen Systemen erfahren. Dabei spielen Medien, die das System der Hörer unmittelbar mit dem der Produzenten koppeln, wie z.B. Fanpost, eine nur geringe Rolle. Einflussreicher auf die Handlungen des Musikers, wenn auch wesentlich komplexer, ist seine Koppelung an das System Geld. Verkaufszahlen und daraus resultierendes Einkommen belegen ihm den Erfolg seiner Handlungen. Er hält sich für verstanden und reagiert mit weiteren, ähnlichen Mitteilungen auf diesen Erfolg. Die Koppelung zweier sozialer Systeme (Musiker und Hörer) über ein drittes (Geldwirtschaft) schränkt allerdings die Möglichkeit des Erfolgs der Kommunikation auf beiden Seiten stark ein. Allein schon die Entscheidung des Hörers, eine CD oder das Video zu kaufen, kann durch Faktoren motiviert sein, die nichts mit der Kommunikation zwischen Hörer und Musiker zu tun haben. Der Besitz der CD kann in einem sozialen System (zu dem der Musiker als Beobachter keinen Zugang hat) z.B. als Zeichen der Teilhabe dienen. Die Kaufentscheidung kann aber auch durch den Preis und die aktuelle Verfügbarkeit der Ware motiviert sein. Problematisch ist auch der Versuch des Musikers, Charts bzw. Hitlisten als Medien des Hörerverhaltens zu deuten. Sie sind Resultate der Auswertungssysteme und machen nicht Anschlusshandlungen der Hörer an Mitteilungen des Musikers beobachtbar. Ein weiteres Medium, über das ein Musiker versuchen kann, Reaktionen auf seine Mitteilungen zu erfahren, ist die Musikpresse. Auch hier koppelt er sich jedoch an ein sekundäres, ebenfalls geschlossenes System, noch dazu an ein anderes Massenmedium, das ebenso wenig unmittelbaren Kontakt zu seinen Lesern hat wie der Musiker zu seinen Hörern.

Den Erfolg einer Mitteilung im Verbreitungsmedium Videoclip einzuschätzen ist noch komplexer als den einer Schallaufzeichnung. Trotz der erfolgreichen Einführung der DVD hat der Videoclip als »harte« Ware bis heute keine nennenswerten eigenen Marktanteile erzielt. Entsprechend marginale Bedeutung haben auch journalistische Kritiken von Clips. Den Erfolg des Clips von den Verkaufszahlen der CD abzuleiten, bedeutet unweigerlich die Unsicherheit der Kommunikation noch weiter zu steigern. Das gleiche gilt für die Beobachtung des Airplay. Ob ein Clip in die Heavy Rotation eines Videosenders wie MTV oder VIVA aufgenommen wird, bestimmen zunächst die Programmmacher. Das Publikum bestimmt erst darüber, wie lange der Titel dort bleibt - und zu diesem Publikum gehört nicht nur der Hörer, den sich der Musiker vorstellt, sondern auch die Werbeindustrie, die das Programm finanziert. 
Die Ferne des Musikers (ich gebrauche den Begriff als Platzhalter für das gesamte soziale System) von seinen Hörern macht für ihn die Vorhersehbarkeit ihrer Handlungen unmöglich, sie produziert genau das, was Kommunikation eigentlich einschränken soll: Kontingenz. ${ }^{7}$ Hinzu kommt, dass zwischen den hier erwähnten Systemen keine Reaktion auf einzelne Handlungen des individuellen Musikers prozessiert wird, sondern immer nur eine Reaktion auf das Medium als Ganzes. Verkaufszahlen und Hitparaden belegen den Erfolg der CD oder des Titels, nicht den eines Gitarrenriffs oder einer Gesangsmelodie. Selbst die Kritik in der Musikpresse bewertet ein Stück in der Regel nicht analytisch, sondern z.B. durch Analogien zum Sound anderer Bands oder durch Werturteile, die sich wiederum auf das Ganze beziehen. Einfluss auf die Handlungen des Musikers kann nur seine direkte soziale Umgebung nehmen, d.h. die an der Produktion Beteiligten. Das soziale System der Produktion ist geschlossen, die Hörer sind kein Teil des Systems. Die Geschlossenheit des Systems bewirkt, dass Kommunikation mit dem Zuschauer zwar Thema im System sein kann, nicht jedoch Bestandteil des Systems selbst. Die Handlungen, die zur Produktion des Clips führen - und nur diese sind den Mitteilenden möglich - sind auf keinen Fall mit den Handlungen identisch, die dem Zuschauer auf dem Bildschirm zugänglich sind. Wie der Zuschauer mit einem virtuellen Musiker kommuniziert, operiert das System des Mediums mit einem virtuellen Zuschauer, der aus den Beobachtungen der an der Produktion Mitwirkenden von anderen Medien wie z.B. der Geldwirtschaft, der Musikpresse oder anderen sozialen Systemen konstruiert wird. Die Realität der Produzenten und die Realität der Zuschauer ist an keiner Stelle und zu keiner Zeit deckungsgleich. Videoproduzenten einerseits und Zuschauer andererseits - und das gilt auch für Wissenschaftler beobachten ihre jeweils eigene Realität ohne Kontaktmöglichkeit in einer für beide identischen Realität.

Die Unmöglichkeit zu wissen, was tatsächlich vom Hörer als Mitteilung verstanden wird und woran dessen (über andere Medien) beobachtetes Verhalten tatsächlich anschließt, führt dazu, dass der Musiker sein Mitteilungsverhalten möglichst allgemein formuliert, also Handlungen verwendet, von denen er annimmt, dass sie seinen Adressaten vertraut sind. Zugleich muss er versuchen, sein Video für die Zuschauer mit einem Überfluss an Anschlussmöglichkeiten auszustatten, um sicher zu stellen, dass sich eine mög-

7 In gewissem Maße planbar wird Erfolg nur, wenn ein direkter Kontakt zwischen Hörern und Massenmedium möglich ist, wie z.B. in den Castingsendungen Deutschland sucht den Superstar oder Popstars. 
lichst große Zahl angesprochen fühlt. Allgemeinverständlichkeit ${ }^{8}$ und Informations-Overkill erzeugen Kontingenz und Beliebigkeit. Sie reduzieren die Kontrolle des Musikers über das Anschlussverhalten seines Publikums noch weiter. Aus diesem medial bedingten Zwang zur Häufung von banalen Sinnangeboten ist die von der Schallplattenhülle über Promotion-Fotos bis hin zum Videoclip zunehmende Verbilderung und in Promotion-Texten und Interviews zunehmende Versprachlichung des Systems Musik zu verstehen. Sie sollen den Erfolg von Kommunikation sichern und entfesseln doch eine ganz eigene Dynamik, eine Inflation der Beliebigkeit. Die Kommunikation zwischen Musiker und Publikum ist - gerade wegen der Distanz - sehr tolerant gegenüber dieser Beliebigkeit des Verstehens. Kommunikation bleibt so lange bestehen, wie Zuschauer bzw. Hörer überhaupt noch Mitteilungen wahrnehmen (egal welche) und für Musiker noch eine Anschlusshandlung beobachtbar bleibt: der Kauf bzw. das Airplay des Produkts.

Massenmedien wie die Schallaufzeichnung und der Videoclip haben die Eigenschaft, auf der Seite der Adressaten unabhängig zu sein von der Ausbildung spezialisierter sozialer Systeme, auf die man in unserem Zusammenhang den Begriff der »interpretive communities « (Fish 1980) übertragen könnte. In Konzerten der populären Musik kann sich der Zuschauer noch direkt und synchron zum Geschehen auf der Bühne am Verhalten seiner Umgebung orientieren, tanzen, wenn alle tanzen, träumen, wenn Feuerzeuge geschwenkt werden, Luftgitarre spielen, wenn der Gitarrist ein Solo hat. Die Schallaufzeichnung hat mit der Diskothek immerhin ein soziales System hervorgebracht, das gegenseitige Verhaltensorientierung der Hörer in einem beschränkten Kontext ermöglicht. Videoclips jedoch werden überwiegend allein geschaut bzw. in sozialen Zusammenhängen, die keine Interaktion der Zuschauer zur gegenseitigen Orientierung über das Gesehene untereinander voraussetzen, neben dem heimischen TV-Gerät z.B. auch in Abteilungen für Jugendmode der Kaufhäuser oder in Gaststätten (Altrogge/Amann 1991: 46f., 49). ${ }^{9}$ Während der Videoclip läuft, kann man telefonieren, Hausaufgaben machen oder sich unterhalten. Es finden jedoch selten beobachtbare Anschlusshandlungen statt, die das Verhalten eines anderen Beobachters orientieren oder gar zur Formierung von »Interpretationsgemeinschaften «

8 Allgemeinverständlichkeit bedeutet nicht, dass eine Mitteilung von möglichst vielen »richtig (im Sinne des Musikers) verstanden wird, sondern nur, dass sie überhaupt irgendwie Anschlüsse ermöglicht.

9 Altrogge/Amann (1991: 304) fragten nur nach den Sehgewohnheiten von Musiksendungen. Da sie hierdurch auch Formate wie die ZDF-Hitparade und Formel Eins mit einbezogen, die traditionell ein Familienpublikum hatten, fiel das Ergebnis vermutlich weniger deutlich aus, als wenn sie nach dem Konsum reiner Videoclipsender gefragt hätten. 
führen könnten. ${ }^{10}$ Über Videoclips wird kein obligatorisches Sozialverhalten koordiniert. ${ }^{11}$ Die Zeit der Unterhaltung ist eine Zeit, die der Zuschauer aus der ihn selbst angehenden Zeit ausgliedert (Luhmann 1996: 99). Im Gegensatz zum Medium des Kunstwerks, das zur Auseinandersetzung mit dem Mitteilenden und über die Mitteilung zwingt, involvieren unterhaltende Massenmedien den Zuschauer nur als Beobachter zweiter Ordnung (ebd.: 107). Kommunikation kommt zustande mit dem Einnehmen der Beobachterhaltung, mit der Herstellung von Aufmerksamkeit, die darin besteht, mögliche Anschlüsse an beobachtete Informationen zu formulieren, frei von allen Verpflichtungen und Konsequenzen für das Ich mit Sinn zu spielen und dadurch "Spannung « zu produzieren. Der Beobachter muss auf Kommunikation nicht mit Kommunikation antworten, er ist zu keinem Anschluss verpflichtet. Eher verhindert der Beobachtungskontext - allein vor dem Fernseher im heimischen Wohnzimmer - noch jeden Willen zur Reaktion. In dieser Beziehung sind Clips reinste massenmediale Unterhaltung: von Massen rezipiert, doch für den Einzelnen bestimmt, Unterhaltung durch massenhafte Individualität (vgl. die »einsame Masse « bei Sønstevold/Blaukopf 1968).

Im Spielfilm verfolgt der Zuschauer ein von seiner Realität unabhängiges Operieren von Sinn, reine Fremdreferenz, ohne jede Pflicht zur Selbstreferenz, die ihm in der Alltagsrealität nicht möglich ist, denn dort impliziert jede Beobachtung eine Entscheidung über den eigenen Anschluss, auch das Nicht-Kümmern oder Ignorieren sind Anschlusshandlungen. Sinn, also Anschlussmöglichkeiten, produziert der Filmzuschauer jeweils aus der Sicht des aktuell Handelnden. Da er jedoch überwiegend die Handlungen eines oder mehrerer Protagonisten gezeigt bekommt, entsteht in der Retrospektive der Eindruck der Identifikation mit einer Hauptperson (und zwar derjenigen, deren Handlungen für den Beobachter am meisten Sinn eröffnen).

Videoclips haben im Unterschied zum Spielfilm eine andere Struktur der Fremdreferenz. Wie schon die Schallaufzeichnung operieren sie stark mit dem Thema Du, d.h. mit einem Gegenüber in der Kommunikation, das im geschlossenen System des Mediums allerdings nur Thema, nicht jedoch Ele-

10 Andere Sendeformate dagegen laden - wenn auch nicht zwangsläufig - zur Bildung von temporären Interpretationsgemeinschaften ein: Fußballübertragungen z.B. oder auch Ratespiele. Vgl. Hepp 1998: bes. $155 \mathrm{ff}$.

11 Knapp ein Drittel der Befragten in der Studie von Altrogge und Amman (1991: 177) gab an, sich durch Videoclips über Tanzstile und die entsprechende Szene orientieren zu wollen. Die Orientierung des eigenen Verhaltens an den beobachteten Tanz- oder Kleidungsstilen, ist jedoch fakultativ. Sie führt nicht zur Bildung von interpretive communities, da das entsprechende Verhalten erst nach dem Ende des Clips gezeigt wird und von einem Beobachter daher nicht unmittelbar auf das Video bezogen werden kann. 
ment ist. Dieses Alter-Ego ${ }^{12}$ entsteht durch die Position des Autors im Clip. Der Musiker, dessen Handlungen der Adressat Ego im Video oder im Song beobachtet, wendet sich mit dem »Du« in seinen Songtexten, mit seiner Körpersprache und den entsprechenden Kameraperspektiven an ein Gegenüber. Im Vergleich zum Medium Konzert ist das Medium Schallaufzeichnung vergleichsweise arm an Informationen. Der Hörer kann lediglich die akustische Seite des Musikmachens beobachten, inm fehlen visuelle Informationen (Aussehen, Bewegungen der Musiker, räumlicher Kontext, historischer Kontext) wie auch soziale Informationen, die im Konzert durch die Beobachtung des Verhaltens der anderen Zuschauer möglich sind. Videoclips liefern genau diese Informationen. ${ }^{13}$ In der Mehrzahl ist tatsächlich zumindest ein Musiker wie auch meistens ein Publikum zu beobachten, das als Zuschauer eines Konzerts, aber auch als kleine Gruppe (z.B. als Gang eines Rappers) oder als einzelne Person (z.B. als nicht musizierende Protagonistin) die Position des Adressaten im Clip repräsentiert. Die Darstellung der Interaktion zwischen Musiker und virtuellem Publikum soll dem Beobachter am Bildschirm Möglichkeiten des Anschlusses vorführen. In die beobachtete Fremdreferenz, die Interaktion zwischen anderen, wird als Vorschlag Selbstreferenz, die Interaktion mit dem Beobachter, eingebaut.

Fernsehnachrichten und Werbesendungen, die ebenfalls ihre Zuschauer direkt ansprechen, gehen davon aus, dass ihre Zuschauer einen unmittelbaren Selbstbezug herstellen. Wenn sie nicht nur unterhalten wollen, müssen Sie sich an der Möglichkeit für den Zuschauer orientieren, auf ihre Mitteilungen mit Anschlussverhalten zu reagieren. Der Zuschauer empfindet seine Realität und die der Nachrichten bzw. der Werbung (wenn sie wirkt) als zumindest teilweise deckungsgleich. Der Videoclip und auch die Schallaufzeichnung dagegen behalten die Unverbindlichkeit reiner Fremdreferenz eben gerade dadurch, dass sie den Beobachter direkt ansprechen und inn damit virtuell in ihre Realität einbinden. Der Zuschauer kann sich selbst in einer Position der Fremdreferenz beobachten: Welche Möglichkeiten von Anschlusshandlungen an die konkrete Information $X$ hätte ich im Publikum von Lenny Kravitz, in der Gang von Lil' Kim oder im Bett mit Madonna? Im Rahmen der beobachteten Realität des Massenmediums wird Selbstreferenz eines Ichs möglich, das Teil dieser Realität ist. Mit dem Ende des Clips -

12 Gemeint ist kein Gegenentwurf Egos zu sich selbst, sondern ein Ego, das der Beobachter Ego der Position Alters zurechnet.

13 Altrogge/Amann (1991: 177) berichten z.B. von einer Nutzung von Heavy MetalVideoclips, um mangelnde Textverständlichkeit auszugleichen und um »die bevorzugte Musikgruppe auch agieren zu sehen«. Damit seien Bedürfnisse nach Information über neue Tanzstile und die betreffende Szene verknüpft. 
bzw. mit dem Ende der Autorposition des Musikers und dem Beginn eines »narrativen« Abschnittes (vgl. Pape/Thomsen 1997: 202ff.) - endet auch die Existenz dieses Ichs. Ob der Beobachter zweiter Ordnung aus der Beobachtung von Selbstreferenz in Fremdreferenz Konsequenzen zieht, d.h. sein Verhalten in der Alltagsrealität an die beobachteten Informationen anschließt und eine tatsächliche Selbstreferenz aufbaut, ist ihm freigestellt. Er weiß, dass von der anderen Seite des Mediums niemand von ihm ein beobachtbares Anschlussverhalten erwartet und erwarten kann, durch das Kommunikation aufrecht erhalten wird - abgesehen von seiner Aufmerksamkeit. Die geringe Ausprägung von interpretive communities auf Seiten der Zuschauer stellt inm darüber hinaus auch im Kontext des Publikums in der Regel jegliches Anschlussverhalten frei.

Das Spiel mit Selbstreferenz in einer Aus-Zeit und exterritorialen Realität macht den Clip zu einer problematischen Form der Unterhaltung. Unterhaltung, die Beobachtung von reiner Fremdreferenz, gilt immer nur für die drei Minuten eines Clips, in denen der Zuschauer sich mit dem beobachteten Alter-Ego identifizieren kann. Fühlt er sich nicht angesprochen, wird der Clip als irrelevant oder sogar störend empfunden und seine Aufmerksamkeit wendet sich einer anderen Tätigkeit zu. Aus diesem Grund haben viele Clips Anteile narrativer Abschnitte, die das Alter-Ego verdrängen und dem Beobachter damit die Einnahme der Perspektive der Handelnden - des Musikers z.B. oder eines anderen Protagonisten - ermöglichen. Die vom Standpunkt der Unterhaltung riskante Darstellung eines fiktiven Autors (Luhmann 1996: 106f.) ist jedoch wichtig für die Funktion des Videos. Die Selbstreferenz in Fremdreferenz enthält zwar keine Verpflichtung, sie behält jedoch das Potential, in einem bestimmten Kontext in tatsächliche Selbstreferenz umzuschlagen und zu tatsächlichen Anschlusshandlungen zu führen. In entsprechender Partylaune kann man durchaus durch das Gegenüber im Clip zum Tanzen stimuliert werden. Darüber hinaus ist die Darstellung der Autorposition im Video wichtig für den Eindruck der Authentizität (vgl. ebd.), die für die Ästhetik populärer Musik nach wie vor von großer Bedeutung ist. Allerdings handelt der Autor des Videoclips nicht dokumentarisch, er tritt nicht aus dem Gezeigten heraus, um das Zeigen zu kommentieren, wie z.B. der Produzent einer TV-Dokumentation. Er beschränkt sich auf die Position des Zeigens, des Vor-Machens und kann so Teil der Fiktion bleiben. Die Aufmerksamkeit des Zuschauers bleibt auf das Gezeigte, das »was«, fixiert und schaltet nicht auf die Frage nach dem Machen, dem »wie«, um. Ein solcher Wechsel der Aufmerksamkeit würde die von beiden Seiten unerwünschte Frage nach dem tatsächlichen Autor aufwerfen und die Virtualität der Authentizität aufdecken. 
Videoclips unterscheiden sich auch von Werbung, mit der sie in der älteren Literatur so häufig gleichgesetzt werden. Werbung braucht tatsächlichen Selbstbezug, um erfolgreich sein zu können. Clips kommen auch dann an, wenn der Selbstbezug ausschließlich in einer anderen Realität stattfindet, d.h. sie fordern nicht zum Kauf einer CD auf, implizieren kein besseres, sicheres, sozial anerkanntes Leben durch ihren Erwerb. Das Boasting des Rappers mit Symbolen seines Reichtums führt dem Zuschauer geradezu vor, dass der Kauf nicht dem Käufer, sondern dem Musiker ein gutes Leben ermöglicht. Das Argument für den Kauf muss der Zuschauer in sich selbst oder in seinem sozialen Umfeld finden, z.B. im Wunsch nach Wiederholung eines Erlebnisses (durch das Zuschauen oder beim Zuschauen) oder nach dem Besitz eines »Statussymbols«.

Es bleibt die Frage nach dem Sinn der Analyse und hermeneutischen Deutung von Videoclips. Das Massenmedium Notendruck brauchte und braucht auch heute noch notwendig und zwangsläufig interpretive communities oder genauer soziale Systeme mit Musikern und Musikwissenschaftlern, die Sozialverhalten im Anschluss an das Medium koordinieren. Sie sind hoch spezialisiert und letztlich die einzigen, für die Notation Information produziert und Anschlusshandlungen ermöglicht. Laien sind auf die Beobachtung ihres Verhaltens angewiesen, um Zugang zu Musik zu erlangen. Die analytische Arbeitshaltung der Musikwissenschaft ergibt sich aus der sachlichen Dimension des Mediums. Jeder einzelne Notenkopf, jede einzelne Pause als Fleck von Tinte oder Druckerschwärze wird von Musikern als Mitteilung des Komponisten und Aufforderung zu Anschlusshandlungen verstanden. Was für den Musiker gilt, muss auch für den Musikwissenschaftler gelten, der diese Aufforderung mit sprachlichem Verhalten beantwortet. Da die Handlungen des Komponisten und seine internen Repräsentationen des Verhaltens von Musikern (oder wie immer er auch Musik konstruiert) unzugänglich sind, ist Interpretation unabdingbar und zwar Interpretation, die um einen Konsens über die »richtige« Nachgestaltung der Interaktion des Komponisten mit dem Medium durch Interaktion des Musikers mit dem Medium ringt. Das Medium zwingt zur Bildung sozialer Systeme, zur Formulierung von Teilen und zur Bildung von Konsens über die Angemessenheit einer spezifischen Anschlusshandlung.

Dieser Zwang zur Konsensbildung (der kein Konsenszwang ist) ist dem Medium Videoclip fremd. Es braucht aufgrund seiner Allgemeinverständlichkeit - es beschreibt ja Handlungen, die direkt beobachtbar sind - nicht notwendig soziale Systeme, die ihrer Beschreibung durch ein weniger spezialisiertes Medium dienen. Eine Wissenschaft, die sich mit Videoclips aus- 
einandersetzt, trifft demnach auf ganz eigene Meinungen von Beobachtern außerhalb des Wissenschaftssystems, die ihren Wahrheitswert aus der eigenen empirischen Erfahrung erhalten. Dem Einwand »Ich habe das aber ganz anders gesehen « hat Wissenschaft kaum Autorität und noch weniger Wissen oder Wahrheit entgegenzusetzen, da sie keine gesamtgesellschaftliche Legitimation zur Interpretation und Beschreibung von Popkultur hat (vgl. Helms 2002).

Das Fehlen ausgeprägter interpretive communities verhindert die Ausbildung eines Konsenses über standardisierte Zeichensysteme, die dem Medium als eigen zugeschrieben werden können. Zeichen, auf die Videoanalysen ihre Exegese gründen, stammen alle aus anderen Medien, die bei der Übertragung auf das Medium Videoclip unweigerlich ihren unbedingten Zeichencharakter verlieren müssen. Der einsame Beobachter ist frei, den Clip von Lil' Kim als ordinäre Zurschaustellung weiblicher Reize und Angeberei mit Luxusgütern oder eben als das ironisch gebrochene Spiel mit den entsprechenden Zeichen zu verstehen (vgl. den Beitrag von Sonja Henscher in diesem Band). Auch das soziale System, dessen Handlungen die Form des Mediums konstituiert, das System der Produzenten, hat keine Autorität zur Festlegung von Sinn bzw. Bedeutungen. Diese hätte es nur, wenn es Teil des sozialen Systems wäre, in dem das Publikum Sinn produziert, wenn Musiker in direkte Kommunikation mit ihren Hörern und Zuschauern treten könnten.

Das System der Zuschauer wird nicht, wie die Systeme von Musikern und Musikwissenschaftern, durch Konsensbildungsprozesse zusammengehalten. Es ist von einer Einigung auf eine wahre Beschreibung nicht abhängig. Wenn soziale Systeme bei der Betrachtung von Videofilmen durch den vereinzelten Zuschauer überhaupt eine Rolle spielen, dann vor allem als Fremdreferenz oder Selbstreferenz in Fremdreferenz. Die gezeigten Ereignisse sind in sich sinnvoll, sie brauchen die Selbstreferenz des Beobachters nicht, um Sinn herzustellen. D.h. dem Betrachter des Clips muss sich die Frage nach Bedeutung oder Wirkung überhaupt nicht stellen. Das wissenschaftliche Bemühen mag ihm lächerlich oder unangemessen vorkommen.

Analyse fragt nach den Teilen und kann das Ganze nicht beschreiben; Systeme, die mit Hilfe des Mediums Videoclip kommunizieren, sind dagegen unsensibel gegenüber den Teilen und reagieren eher auf das Ganze. Wenn Clips doch einmal gemeinschaftlich geschaut werden, sind gelegentliche Kommentare wie »Guck dir das an!« oder »Mach das aus! « kaum aussagekräftig genug, um Teile und aus Teilen Zeichen zu generieren. Das soziale System der Zuschauer bildet vielleicht einen Konsens über die Bewertung des ganzen Clips - ob dieser in Teile, Ebenen, Variablen oder Zeichen geteilt und an diesen sein Wert festgemacht werden kann, fragt es nicht. 
Durch die Ferne der Systeme von Musikern und Publikum kann sich auch zwischen ihnen Kommunikation nur auf das Ganze, nicht jedoch auf die Teile beziehen. ${ }^{14}$ Die Analyse von Videoclips bleibt auf das System der Wissenschaft beschränkt, die ihre Methoden aus der Beschäftigung mit Kunstwerken auf Massenmedien zu übertragen versucht. Ihre Ergebnisse haben keine Relevanz für das System der nicht-wissenschaftlichen Zuschauer.

Die Vorstellung vom Werk erlaubte es dem Nachdenken über Musik zur Wissenschaft zu werden. Im Bezug auf das Werk ist es der Musikwissenschaft möglich, einen Konsens über wahr oder unwahr einer Aussage herzustellen. Videoclips sind jedoch keine Werke. Die Handlungen ihrer Macher sind offensichtlich (auch wenn sie nicht das Machen zeigen), ihr Schöpfer ist ein komplexes soziales System. Aufgrund der Eigenschaften des Mediums Videoclip, aber auch aufgrund ihrer mangelnden Autorität für die Systeme der Zuschauer wie auch der Produzenten, ist der Musikwissenschaft die Möglichkeit der Unterscheidung zwischen wahr und unwahr genommen. An ihre Stelle tritt die Einordnung zwischen die Pole richtig oder falsch. Diese Kategorie ist ausschließlich auf das Verhalten des Zuschauers bezogen. Es kann nicht mehr darum gehen, Konsens darüber herzustellen, wie der Autor etwas gemeint hat oder gemeint haben könnte, sondern was der Zuschauer meinen könnte, dürfte, sollte, damit seine Meinung »richtig « ist. Dieses Richtig oder Falsch ist jedoch immer nur im sozialen Kontext des Beobachters zu verstehen. Es läuft - eine große Zahl der wissenschaftlichen Beiträge zum Videoclip und auch einige Artikel dieses Bandes zeigen es deutlich letztendlich auf eine moralisch-ethische Bewertung zu. Mit dem Verlust der Wahrheit des autonomen Werkes nähert sich wissenschaftliche Ästhetik zwangsläufig wieder der Ethik an. In der Tat scheint das gesellschaftliche Interesse an einer Institution zur Folgeabschätzung massenmedialer musikalischer Unterhaltung größer zu sein als an einer Institution zur Analyse von Form und Inhalt. Damit kommt Musikwissenschaft wieder dort an, wo sie mit der Staatstheorie Platos und Aristoteles' in der griechischen Antike ihren Ausgang nahm. Im Gegensatz zu den antiken Philosophen, die Musik im überschaubaren Rahmen einer idealen griechischen Polis beschrieben, muss es heutigen Autoren als Naivität angerechnet werden, wollten sie ihre Urteile über Musik absolut setzen, ohne die eigene Autorität zu hinterfragen. Es

14 Aus diesem Grund sind Konzerte mit ihrer - wenn auch eingeschränkten - Möglichkeit des direkten Kontaktes trotz und gerade wegen der Erfindung von Schallplatte und Videoclip notwendig. Aus demselben Grund wurde auch die Erfassung des Airplay einzelner Titel notwendig, nachdem die Single fast verschwunden war und nur noch LPs bzw. CDs verkauft wurden. Die Verkaufszahlen von LPs ermöglichen keinen Rückschluss auf besonders beliebte Titel. 
ist notwendig, ja unverzichtbar, eine Sensibilität für die eigene Beobachterposition zu entwickeln, wie auch ein Bewusstsein für die Komplexität des Zusammenspiels sozialer und psychischer Systeme - sowohl für die Systeme, die beschrieben werden, als auch für solche, in deren Kontext Beschreibungen stattfinden.

\section{Literatur}

Altrogge, Michael (2000). Tönende Bilder. Interdisziplinäre Studie zu Musik und Bildern in Videoclips und ihrer Bedeutung für Jugendliche. 3 Bde. Berlin: Vistas.

Altrogge, Michael / Amann, Rolf (1991). Videoclips - die geheimen Verführer der Jugend? Ein Gutachten zur Struktur, Nutzung und Bewertung von Heavy-MetalVideoclips. Berlin: Vistas.

Bullerjahn, Claudia (2001). Grundlagen der Wirkung von Filmmusik. Augsburg: Wißner.

Dahlhaus, Carl (1977). Grundlagen der Musikgeschichte. Köln: Gerig.

Ehrenfels, Christian von (1967). »Über Gestaltqualitäten. «In: Gestalthaftes Sehen. Ergebnisse und Aufgaben der Morphologie. Hg. v. Ferdinand Weinhandl. Darmstadt: Wissenschaftliche Buchgesellschaft, S. 11-43. [1. Ausg. in: Vierteljahresschrift für wissenschaftliche Philosophie 14. 1890. 3].

Fish, Stanley Eugene (1980). Is There a Text in This Class? The Authority of Interpretive Communities. Cambridge, Mass., London: Harvard University Press.

Gadamer, Hans-Georg (1986). Wahrheit und Methode. Grundzüge einer philosophischen Hermeneutik (= Gesammelte Werke 1). Tübingen: Mohr (5. erw. Aufl.).

Großmann, Rolf (1997). „Konstrutiv(istisch)e Gedanken zur >Medienmusik «« In: Medien-Musik-Mensch. Neue Medien und Musikwissenschaft. Hg. v. Thomas Hemker und Daniel Müllensiefen. Hamburg: von Bockel, S. 61-78.

Helms, Dietrich (2002). »Musikwissenschaftliche Analyse populärer Musik?«In: Musikwissenschaft und populäre Musik. Versuch einer Bestandsaufnahme. Hg. v. Helmut Rösing, Albrecht Schneider und Martin Pfleiderer (= Hamburger Jahrbuch für Musikwissenschaft 19). Frankfurt/M. u.a.: Lang, S. 91-103.

Helms, Dietrich (2003). »Auf der Suche nach einem neuen Paradigma: Vom System Ton zum System Sound. «In: Pop Sounds. Klangtexturen in der Pop- und Rockmusik. Basics - Stories - Tracks. Hg. v. Thomas Phleps und Ralf von Appen (= texte zur populären musik 1). Bielefeld: Transcript, S. 197-228.

Hepp, Andreas (1998). Fernsehaneignung und Alltagsgespräche. Fernsehnutzung aus der Perspektive der Cultural Studies. Opladen: Westdeutscher Verlag.

Jauß, Hans Robert (1991). »Rückschau auf die Rezeptionstheorie. Ad usum Musicae Scientiae. «In: Rezeptionsästhetik und Rezeptionsgeschichte in der Musikwissenschaft. Hg. von Hermann Danuser und Friedhelm Krummacher. Laaber: Laaber, S. 13-36.

Luhmann, Niklas (1984). Soziale Systeme. Grundriß einer allgemeinen Theorie. Frankfurt/M.: Suhrkamp.

Luhmann, Niklas (1996). Die Realität der Massenmedien. Opladen: Westdeutscher Verlag (2. erw. Aufl.).

Luhmann, Niklas (1999). Die Kunst der Gesellschaft. Frankfurt/M.: Suhrkamp.

Pape, Winfried / Thomsen, Kai (1997). »Zur Problematik der Analyse von Videoclips. «In: Step Across the Border. Neue musikalische Trends - neue massen- 
mediale Kontexte. Hg. v. Helmut Rösing (= Beiträge zur Popularmusikforschung 19/20). Karben: Coda, S. 200-219.

Roth, Gerhard (1997). Das Gehirn und seine Wirklichkeit. Kognitive Neurobiologie und ihre philosophischen Konsequenzen. Frankfurt/M.: Suhrkamp.

Sønstevold, Gunnar / Blaukopf, Kurt (1968): Die Musik der »einsamen Masse«: Ein Beitrag zur Analyse von Schlagerschallplatten (= Schriftenreihe Musik und Gesellschaft 4). Karlsruhe: Braun.

Schläbitz, Norbert (1997). »Medien - Musik - Mensch.«In: Medien-Musik-Mensch. Neue Medien und Musikwissenschaft. Hg. v. Thomas Hemker und Daniel Müllensiefen. Hamburg: von Bockel, S. 11-38.

\begin{abstract}
This paper describes music videos as highly complex processes of communication on the theoretical basis of Niklas Luhmann's theory of social systems. The application of the analytical approach of musicology towards music videos is questioned as it was developed for a mass medium that indeed needed verbal and musical interpretation: notation. Videos, however, are obvious, they make sense to almost everybody and they are independent of »interpretive communities« (Fish) as mediators of »meaning «. The social systems of producers and consumers of videos are closed and without any possibility of direct interaction. Musicians have to gather the success of their messages through a third, intermediate medium like money or journalism. For musicians on the one hand predicting consumer behaviour becomes an impossible task, the audience, on the other hand, is free to create meanings of its own, which are - lacking strong and influential interpretive communities - highly individualized. Communication through and about videos concerns the medium as a whole, not parts of it. The audiences' approach towards the medium is synthetic rather than analytical.
\end{abstract}

